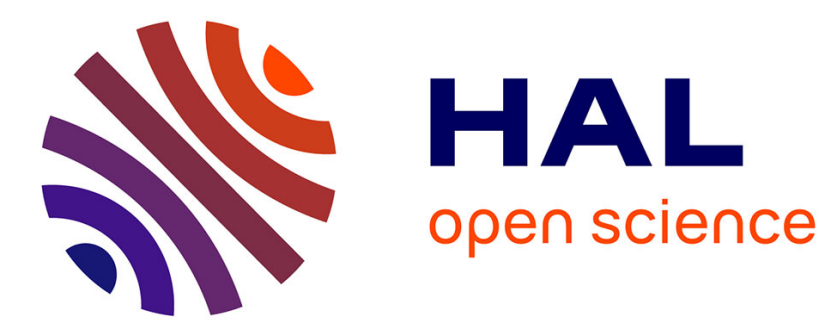

\title{
Physical Activity and Sarcopenia
}

Fabien Pillard, Dalila D. Laoudj-Chenivesse, Gilles Carnac, Jacques Mercier, Jacques Rami, Daniel Rivière, Yves Rolland

\section{To cite this version:}

Fabien Pillard, Dalila D. Laoudj-Chenivesse, Gilles Carnac, Jacques Mercier, Jacques Rami, et al.. Physical Activity and Sarcopenia. Clinics in Geriatric Medicine, 2011, 27 (3), pp.449-470. 10.1016/j.cger.2011.03.009 . hal-02540902

\section{HAL Id: hal-02540902 \\ https://hal.umontpellier.fr/hal-02540902}

Submitted on 12 Apr 2020

HAL is a multi-disciplinary open access archive for the deposit and dissemination of scientific research documents, whether they are published or not. The documents may come from teaching and research institutions in France or abroad, or from public or private research centers.
L'archive ouverte pluridisciplinaire HAL, est destinée au dépôt et à la diffusion de documents scientifiques de niveau recherche, publiés ou non, émanant des établissements d'enseignement et de recherche français ou étrangers, des laboratoires publics ou privés. 


\section{Physical Activity and Sarcopenia}

Fabien Pillard, MD, PhD ${ }^{a, b, c, *}$, Dalila Laoudj-Chenivesse, $\mathrm{PhD}^{\mathrm{d}}{ }^{\mathrm{e}}$, Gilles Carnac, PhD ${ }^{d}$, Jacques Mercier, $M D, P h D^{d, e, f}$, Jacques Rami, PhD ${ }^{a, b, c}$, Daniel Rivière, $M D, \mathrm{PhD}^{\mathrm{a}, \mathrm{b}, \mathrm{c}}$, Yves Rolland, $M D, P h D^{g, h, i}$

\section{KEYWORDS}

- Exercise prescription • Exercise intensity • Training

Multiple factors and mechanisms contribute to the age-related impairment of the function and mass of the skeletal skinned muscles that indicates the diagnosis of sarcopenia. Recent longitudinal aging studies suggest that sarcopenia may be secondary to muscle weakness in the elderly (see Refs. ${ }^{1,2}$ for reviews). These recent studies, as well as the work of a research group on sarcopenia 10 years previously, ${ }^{3}$ also demonstrated that muscle strength, but not muscle mass, is independently associated with lower extremity performance, another factor for disability among older persons. As exercise training enhances muscle mass and function in nonelderly subjects and as lack of activity (or inactivity) is an important contributor to loss of muscle mass and strength at any age, ${ }^{4-7}$ it is of interest to examine whether physical activity, a functional therapy and a modifiable lifestyle behavior, could be proposed in treatment and prevention of sarcopenia, which is in part a functional disease. In the present article the authors first briefly show how physical activity may alter muscle properties in sarcopenia, then examine its evidence-based clinical effect on sarcopenia. The principles governing the prescription of physical activity for subjects with sarcopenia are also

The authors have no conflicts of interest to report.

a Respiratory Exploration Department and Sports Medicine Department, Larrey University Hospital, 24 chemin de Pouvourville, 31059, Toulouse CEDEX 9, France

b Physiology Department, Medical University of Toulouse III, France

c French National Institute for Health and Medical Research (INSERM), Obesity Research Unit U858 - I2MR, Toulouse, France

d French National Institute for Health and Medical Research (INSERM), ERI 25, Montpellier 34000, France

e Medical University of Montpellier, EA-4202, Montpellier, France

${ }^{f}$ University Hospital of Montpellier, France

9 National Institute for Health and Medical Research (INSERM), U558, Toulouse, France

$\mathrm{h}$ Medical University of Toulouse III, France

i Department of Geriatric Medicine, University Hospital of Toulouse, France

* Corresponding author. Service d'Exploration de la Fonction Respiratoire et de Médecine du Sport, CHU Larrey, 24 chemin de Pouvourville, 31059, Toulouse CEDEX 9, France.

E-mail address: fpillard@hotmail.com 
discussed. The factors that can be modified by physical training and the principles on which prescription should be based are summarized in Fig. 1.

\section{PHYSICAL ACTIVITY FOR SARCOPENIA: FROM HUMAN TO CELL}

Age-related changes in skeletal skinned muscle can largely be attributed to the interaction of factors affecting neuromuscular transmission, muscle (and tendon) architecture, fiber composition, excitation-contraction coupling, and metabolism. Before aging exerts its influence, muscle fibers display plasticity of their biochemical and morphologic properties when they are exposed to different functional demands. Throughout the human aging process, the loss of strength in old age is predominantly accounted for by reduced muscle mass and myofibrillar protein content in consequence of a combination of progressive fiber loss and fiber atrophy. The aging process is often characterized by investigators as a selective loss of fast-twitch fibers in muscle. ${ }^{8}$ However, when electrophoretic and not histochemical identification of muscle fiber phenotype was performed, other studies indicated that the distribution of fiber types is relatively stable across the aging part of the life span, in particular because of the identification of a high percentage of hybrid fibers (coexpressing more than one myosin heavy chain isoform). ${ }^{9}$ However, despite aging, sarcopenia, and significant functional alterations of the overall muscle, the contractile function of surviving fibers may be preserved in older humans as suggested by the upregulation of basal levels of some myogenic regulation factors (MRFs), ${ }^{10-12}$ and by the link between upregulation and the degree of sarcopenia in rodents. ${ }^{13}$ These findings suggest that surviving fibers are still prone to plasticity in response to different functional demands such as physical exercise; this response helps elderly muscle to compensate to partially correct muscle size deficit in an attempt to maintain optimal force-generating capacity. ${ }^{14}$ Regular physical activity can thus partially correct the acceleration of sarcopenic progression related to inactivity and poor nutrition. ${ }^{15}$

From an overall viewpoint, the quantitative aspect of sarcopenia is attributable to an imbalance between protein synthesis and degradation or between apoptosis and regeneration processes, or both. The influence of physical activity in sarcopenic muscle can be described in relation to several of the factors acting on muscle in age-related imbalance processes.

\section{Effect of Physical Activity on Muscle Anabolism in Elderly Subjects}

A key intracellular pathway that coordinates signals in the regulation of muscle protein synthesis is the mammalian target of rapamycin (mTOR), which plays a key regulatory role in the regulation of translation initiation. ${ }^{16}$ Following acute exercise in animals and young humans, components of the mTOR pathway are rapidly upregulated (see Ref. ${ }^{17}$ for review, and Ref. ${ }^{18}$ ), but little is known of changes in mTOR activity and total protein content with chronic exercise. On the other hand, there are only indirect data suggesting positive mTOR signaling mechanisms in aged human skeletal muscle following exercise. ${ }^{19,20}$ A reduction in myostatin gene expression (a negative regulatory factor of skeletal muscle development) in older as well as in young subjects could also influence anabolic response following exercise in older persons. ${ }^{12}$ Serum myostatinimmunoreactive protein levels have been found to be higher in 60- to 75-year-old men and women than in younger 19- to 35-year-olds, and were inversely correlated

with muscle mass corrected for height. ${ }^{21}$ Whatever the pathway, Kosek and colleagues $^{22}$ suggested that the effective, but lower, anabolic muscle response in older than in young men after a 16-week resistance training program, 3 days a week, was not explained by age-related variation in the expression of myogenic 


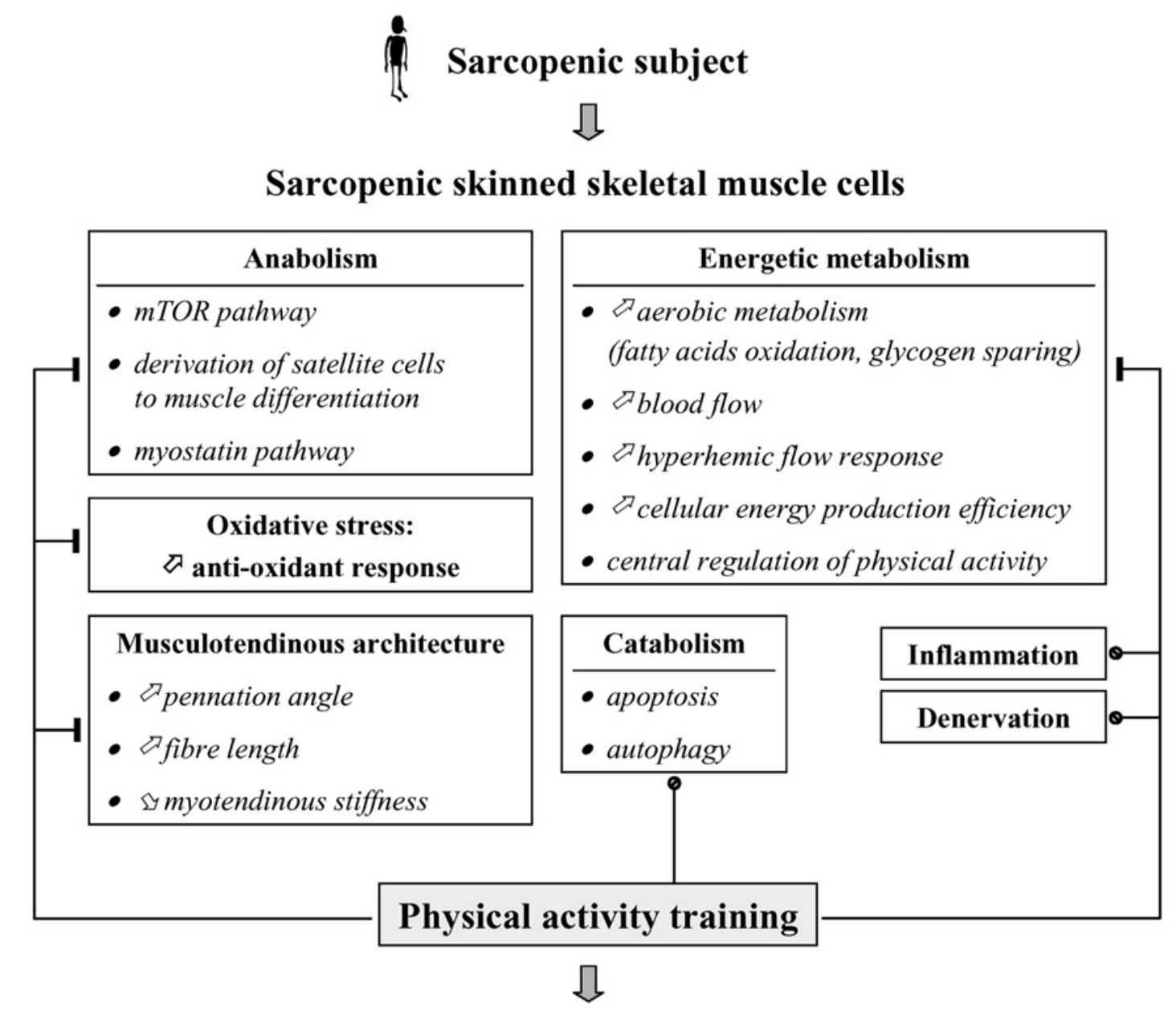

\section{Prescription of physical activity; defining:}

- the drug, i.e. the physical activity agent

- the dose of physical activity that must be delivered (intensity $\times$ time)

- the frequency of the administration of physical activity

- the context of the administration of physical activity (who monitors PA sessions?)

$\left.\begin{array}{|l|c|}\hline \multicolumn{2}{|c|}{\text { Recommended prescription }} \\ \text { - resistance training (and eccentric exercises) } \\ \text { - endurance training } \\ \text { - (low intensity occlusion training) }\end{array}\right\} \begin{gathered}\text { Physically active lifestyle } \\ \text { and codified exercise }\end{gathered}$

Evidence based epidemiological effect of physical activity training on sarcopenia: it's never too late!

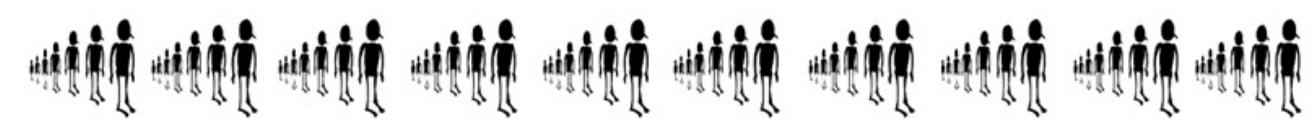

Fig. 1. Summary of the association between sarcopenia and physical activity training. Positive modulation; — negative modulation. 
regulation factors. One explanation was that the RNA-to-protein ratio is higher in old than in young muscles, suggesting a decrease in translational efficiency over the life span. Finally, transcription-mediated effects of physical activity leading to satellite cell activation and proliferation factors could be useful to divert satellite cells to muscle differentiation and not adipocyte differentiation, ${ }^{23}$ because failure to express the transcription factors that direct muscle mesenchymal precursors into fully differentiated functionally specialized cells may be responsible for their phenotypic switch into the adipogenic lineage (see Ref. ${ }^{24}$ for review).

Although both young and elderly humans show an increase in some muscle factors associated with proliferation and differentiation, and a reduction in myostatin gene expression following exercise, the quantitative anabolic response to resistance exercise (protein mass) is lower in the elderly than in the young. Some investigators consider that this could be linked with the opposing and higher expression of proteolytic regulators (see Ref. ${ }^{25}$ for review), whereas according to other investigators upregulation of atrophy gene expression does not seem to be involved in the sarcopenia process. ${ }^{26}$ When interpreting discrepancies of results between investigators, one should bear in mind that both animal and human studies are performed with the common aim of identifying a strategic axis for the management of sarcopenia in humans. Increased apoptosis is another and negative process that has been identified as a possible pathway leading to the development of sarcopenia; as a countermeasure against apoptosis, it has been suggested that increased chronic activity attenuates apoptotic signaling and may reduce sarcopenia in rats. ${ }^{27,28}$ In rodent skeletal muscle, exercise associated with mild caloric restriction may also attenuate the age-related impairment of autophagy (a process of degradation of cellular constituents, that when controlled could be a useful mechanism to combat cell damage and death). ${ }^{29}$

Upstream of the intracellular pathways coordinating muscle protein anabolism in older subjects, some hormones might influence this response of muscle to exercise. Wilkes and colleagues ${ }^{30}$ recently suggested that blunting of insulin inhibition of proteolysis in legs of older subjects may contribute to age-related sarcopenia, and that this effect may be mediated through blunted Akt-PKB activation. As exercise training enhances insulin sensitivity in older subjects, ${ }^{31}$ and according to the authors' previous hypothesis may have a positive effect on mTOR signaling mechanisms, it may also have a positive effect on the sarcopenic process by enhancing insulin inhibition of proteolysis. Androgenic response to exercise has also been hypothesized as a hormonal mechanism explaining muscle response to exercise. In a recent study, Ahtiainen and colleagues ${ }^{32}$ demonstrated in older men that a 21-week long program of strength, endurance, or combined training specifically increased strength and muscle mass as well as aerobic capacity according to the training method, but no significant relationship was shown between these functional adaptations and androgenic response, that is, serum testosterone or cytoplasmic androgen receptor (AR) concentration measured 4 to 5 days after the last training session. This result is in contradiction with the association between androgenic and functional clinical responses observed after exercise and described in other studies ${ }^{33-35}$ but, unlike Ahtiainen's study, these were not performed in elderly subjects and the biological response was evaluated very shortly after the stimulation by muscular exercise. Moreover, as suggested by these investigators, the testosterone effect may be affected by nongenomic signaling mechanisms through transmembrane G-protein-coupled receptors and linked to an increase in free $\mathrm{Ca}^{2+}$ and activation of mitogen-activated protein kinase, ${ }^{36}$ which are signaling pathways in the development of skeletal muscle hypertrophy. ${ }^{37}$ Lastly, the subjects evaluated in the study of Ahtiainen and colleagues had normal plasma testosterone concentrations before starting the physical activity 
program, so the potential variation of this biological parameter in relation to baseline level was smaller. Physical activity could thus have a favorable effect on the "muscle testosterone axis" in subjects with low plasma levels of this hormone, as does testosterone supplementation in the same subjects. Even according to this hypothesis, exercise-mediated androgenic pathway activation may not sufficiently explain the effect of exercise on functional parameters, as there are conflicting and inconclusive results on the effectiveness of testosterone therapy on muscle mass and muscle strength in the elderly, even in hypogonadal subjects. However, no study has used androgen supplementation combined with exercise training to examine how these two treatment methods could each potentialize the other (see Ref. ${ }^{38}$ for review). Such a synergistic effect between exercise and hormone replacement therapy (HRT) has been suggested for estrogen replacement therapy in a randomized controlled trial in middle-aged women in early menopause: additional exercise resulted in additive or even synergistic effects of HRT on muscle (evaluated by vertical jumping height and relative proportion of fat within the muscle compartment). ${ }^{39} \mathrm{~A}$ third "hormonal" response of muscle to exercise training relates to the insulin-like growth factor 1 (IGF-1) pathway. IGF-1 is a potent mitogen and anabolic agent that plays an important role in the growth of various body tissues, including skeletal muscle. The decline of circulating IGF-1 levels with advancing age is also related to loss of muscle mass and strength. Strength training has been reported to increase the skeletal muscle fraction of IGF-1 protein and muscle IGF-1 mRNA levels, that is, the noncirculating fraction of this factor, even in the elderly. ${ }^{40,41}$ It has been suggested that IGF-1 gene polymorphisms influence muscle phenotypic responses to strength training in older men and women, ${ }^{42}$ which could explain why the phenotypic and functional effects of such training on muscle differ between studies.

As a nonspecific cellular pathway and in addition to contractile activity, feeding increases the rate of protein synthesis compared with the unfed state. This process appears to be mainly caused by increased amino acid availability (particularly essential amino acids) rather than feeding-induced increase in insulin concentrations ${ }^{43}$ (see the article Nutrition and Sarcopenia by Volpi and colleagues elsewhere in this issue of the journal).

\section{Effect of Physical Activity on Muscle Energetic Metabolism in Elderly Subjects}

Whether or not one considers that the sarcopenic process may be characterized by selective atrophy of type II muscle fibers, both type I and type II fibers are affected by metabolic dysfunction in the context of sarcopenia.

In healthy older adults, there is evidence of mitochondrial impairment and muscle weakness, but functional indicators of impairment, such as phosphodiester enhancement in muscle, can be reversed and the transcriptome enhanced, resulting in improvement of the sarcopenic muscle phenotype when subjects are physically active. ${ }^{44-47}$ What remains less clear is whether the decline in skeletal muscle mitochondrial oxidative capacity is purely a function of the aging process, or whether the sedentary lifestyle of the elderly subjects studied acts as a confounder in the association between these two factors.

As oxygen availability for mitochondria is a potential limiting factor of aerobic metabolism, reduced muscle capillarity, decreased maximal blood flow, slower hyperemic flow response, ${ }^{48,49}$ arterial stifness, ${ }^{50}$ and disruption of the microvascular endothelium ${ }^{51}$ have been reported in some studies in sedentary older people, but not in all. Exercise training can improve these functions and so could limit at cellular level the mitochondrial dysfunction in sarcopenic muscle. Finally, Chow and colleagues $^{52}$ highlighted that exercise training increased spontaneous physical activity 
(as measured with infrared photocell sensors) in a murine model. Despite the fact that this model was murine and that the rodents were not old, this change could have important implications for aging, because it supports the notion of a relation between mitochondrial function and spontaneous physical activity, and suggests that exercise training may be a viable approach to interruption of the vicious cycle of aging.

When muscular oxidative capacity drops, anaerobic metabolism is more able to sustain energy (adenosine triphosphate [ATP]) production, in particular when exercise intensity increases as in resistance exercise: glycogen then becomes an essential substrate for energy production while fatty acid use decreases. Looking beyond the defect in mitochondrial function, the decrease in resting muscle glycogen concentration after use is not favorable to resistance exercise in older humans. As previously stated, endurance training can reverse mitochondrial dysfunction and so contribute to reduce glycogen depletion because it enhances fatty acids oxidation, making glycogen available for resistance exercise. ${ }^{53}$ Notwithstanding the restoration of oxidative metabolism and the consequent sparing of glycogen, energy supply is limited by the availability of substrates. Fatty acids, proteins, and carbohydrates can be oxidized by muscle, but fatty acids are preferential substrates until the energy output supplied matches with the energy output of muscular contraction, which occurs at low to moderate intensities of endurance exercise. ${ }^{54}$ Sufficient fatty acid oxidation is also of interest because excess fatty acids can limit glycolysis through the Randle cycle ${ }^{55}$ and so supply energy for high-intensity exercise (relative to the maximal intensity). It has been suggested that the availability of muscle carnitine (a substrate for carnitine palmitoyl-transferase that transports acetyl-coenzyme $A$ into mitochondria) may limit fatty acid oxidation. As there seems to be a negative correlation between advancing age and muscle carnitine levels, carnitine deficiency may therefore contribute to geriatric frailty (see Ref. ${ }^{56}$ for review). However, endurance training can reverse carnitine dysfunction in humans, as described by Lanza and colleagues, ${ }^{31}$ who showed in young (18-30 years old) as well as in old subjects (59-76 years old) that endurance training was associated with better ATP production through the carnitine pathway and increased muscular oxidative capacity. In rats, upregulation of uncoupling protein 3 (UCP3) in aged muscle also limited ATP-generating capacity of the fiber. ${ }^{57}$ Exercise training could be suggested as a countermeasure to restore cellular energy production efficiency, as training decreased UCP3 protein levels in young untrained subjects. ${ }^{58}$ This last assumption needs to be tested for sarcopenia in elderly individuals. Training also elicits (restores) UCP3 content in limb muscle of patients with chronic obstructive pulmonary disease, and it has been hypothesized that UCP3 may protect muscle against lipotoxicity (also detrimental to muscle function in sarcopenia). ${ }^{59}$

Another consequence of the mitochondrial dysfunction could be the alteration of the central regulation of physical activity (see Ref. ${ }^{8}$ for details). It is has been suggested that spontaneous physical activity is regulated by hypothalamic centers in response to signals from the peripheral tissues, especially from skeletal muscle mitochondrial ATP. If mitochondrial ATP production in peripheral skeletal muscle declines, thus downregulating activity levels via efferent sympathetic or chemical signals, spontaneous activity would lessen with age in response to declining mitochondrial function in peripheral tissue. Moreover, it has been suggested that reduced spontaneous activity and other unknown regulatory factors interact to reduce the cognitive phenomenon of "motivation" in older persons to engage in voluntary physical activity. According to this model, both spontaneous and voluntary physical activities decrease with age as a result of the alteration of mitochondrial ATP production. Exercise training could then stimulate spontaneous physical activity and motivation in the elderly through its positive effect on the mitochondrial function. 


\section{Effect of Physical Activity on Oxidative Stress in Elderly Subjects}

The level of oxidative stress imposed on cells is influenced by two fundamental biological processes: increased generation of reactive oxygen species (ROS) and decreased generation of antioxidant defense. During aging, as in exposed young subjects, increased oxidative stress in skeletal muscle leads to the accumulation of damaged proteins, which are not properly eliminated, aggregate, and in turn impair proteolytic activities. ${ }^{60}$ Myocytes, satellite cells (SCs), ${ }^{61}$ neuromuscular junction, and mitochondria ${ }^{62}$ are damaged by oxidative stress, and have been depicted as potential mechanisms of sarcopenia. Mitochondrial ROS production is a factor that could be implicated in the adipogenic conversion of SCs. ${ }^{24}$ An association between skeletal muscle weakness and oxidative stress has been confirmed in a cohort study conducted over 36 months in a large $(\mathrm{N}=545)$ sample of American women older than 65 years (the Women's Health and Aging Study I). After adjusting for confounding variables, serum protein carbonyls (markers of oxidative damages to proteins) were associated with a decline in walking speed over 36 months and with incident severe walking disability (hazard ratio 1.42, 95\% confidence interval 1.02-1.98). ${ }^{63}$

Mitochondria are damaged by ROS but also produce them, leading to an adaptive response that subsequently increases stress resistance. This response is assumed to reduce oxidative stress in the long term when in contact with ROS. This type of retrograde response has been named mitochondrial hormesis or mitohormesis, and may in addition be applicable to the health-promoting effects of physical exercise in humans. ${ }^{64}$ The challenge is then to stimulate mitohormesis against sarcopenia by exercise, as this alters mitochondrial function. Although gene expression of some antioxidant enzymes can be enhanced after an acute bout of exercise, the consecutive increase in de novo protein synthesis of an antioxidant enzyme usually requires repeated bouts of exercise. Moreover, according to the concept of "oxidative stress homeostasy," nonexhaustive exercise can induce mild stress that stimulates the expression of certain antioxidant enzymes, although this training-induced adaptation seems to be attenuated by aging. ${ }^{65}$ Targeting oxidative stress with exercise through an antioxidant response seems rational in fighting against age-induced muscle performance impairment that occurs with or without sarcopenia. However, it has recently been suggested that training should be supplemented by exogenous antioxidants to seek the optimal level of defense.

\section{Effect of Physical Activity on Muscle Inflammation in Elderly Subjects}

Inflammation may be a potential factor in the development of sarcopenia through stimulation of proteolytic signaling. Recent evidence indicates that chronic resistance training contributes to the control of locally derived inflammation via adaptations to repeated and acute increases in proinflammatory mRNA within muscle. ${ }^{66}$ Exerciseinduced changes in heat-shock protein $(\mathrm{Hsp})$, responsible for cellular protection during stressful situations, in particular Hsp70 expression, might interfere with the acute-phase reaction (circulating cytokines) after a resistance exercise program. ${ }^{67}$ Endurance exercise may mediate the anti-inflammatory and antiatrophy effects by many routes, including the upregulation of peroxisome proliferator-activated receptor- $\gamma$ coactivator (PGC)- $1 \alpha$ in muscle, downregulation of Toll-like receptors, and enhanced release of proinflammatory interleukin (IL)- 6 by muscles. It has been suggested that mildly elevated expression of PGC-1 $\alpha$ in muscle modulates this inflammatory response not only in the muscle itself but also systemically. ${ }^{68}$ Downregulation of Toll-like receptors itself modulates inflammation (innate inflammation) in aged subjects and indirectly modulates the sarcopenic process because upregulation of 
their activity links with the insulin-resistance process. ${ }^{69}$ It could be surprising to include IL-6 upregulation as an anti-inflammatory process, but IL-6 inhibits the major inflammation pathway through tumor necrosis factor $\alpha$ (TNF- $\alpha$ ) production. ${ }^{70}$ For some investigators, an age-related disruption in the intracellular redox balance appears to be a primary causal factor in producing a chronic state of low-grade inflammation, as ROS appear to function as second messengers for TNF- $\alpha$ in skeletal muscle and also activate nuclear factor $\kappa \mathrm{B}$, which itself upregulates IL- 6 and TNF- $\alpha$ (see Ref. ${ }^{71}$ for review). The effects of exercise training on ROS production could also indirectly modulate the "inflamm-aging." cated that myostatin could positively alter TNF- $\alpha$ production: modulation of myostatin expression by training could then also be identified as a pathway leading to the antiinflammatory effect of training. ${ }^{73}$

\section{Effect of Physical Activity on Muscle Denervation in Elderly Subjects}

Because mobility-limited older adults exhibited impaired activation of the agonist quadriceps and concomitant deficits in torque and power output whereas healthy subjects did not, it has been suggested that neuromuscular activation deficits may contribute to compromised mobility function in older adults. ${ }^{74}$ Aging is characterized by loss of spinal motor neurons due to mechanisms also involved in muscle anabolism and trophicity: apoptosis, reduced IGF-1 signaling, elevated amounts of circulating cytokines, and increased oxidative stress on cells. Therefore, it is of interest to examine whether physical activity could alter the loss of spinal motor neurons. ${ }^{75}$ In rats, age-related denervation occurs before myofiber atrophy, and high amounts of neuromuscular activity may delay the onset of age-related denervation and sarcopenia. ${ }^{76}$ In humans, strength training appears to be an effective countermeasure in elderly individuals even at a very advanced age ( $>80$ years) by eliciting muscle hypertrophy along with substantial changes in neuromuscular function in the first stages of the plasticity response. ${ }^{76,77}$

\section{Effect of Physical Activity on Muscle and Tendon Architecture in Elderly Subjects}

The decrease in muscle volume as a result of aging affects muscle architecture. The decrease in pennation angle and fascicle length during aging has several implications for strength and velocity of contraction. ${ }^{78}$ First, a smaller pennation angle leads to a smaller physiologic cross-sectional area (PCSA, which is the ratio of muscle volume to fascicle length, correlated to force production) and also to a simultaneous increase in the force of the muscle fibers exerted on the tendon, proportional to the cosine of the pennation angle ${ }^{79}$; the latter may compensate in part for the smaller PCSA and so for the decrease in force production. The effect of aging on force production is more pronounced for concentric movements (muscle shortening) than for eccentric movements (muscle lengthening). This phenomenon appears to be linked to stiffer muscle structures (in consequence of an accumulation of noncontractile material in the muscle-tendon unit, which offers increased passive stiffness that contributes to musculotendinous eccentric strength) and prolonged myosin cross-bridge cycles of aged muscles. ${ }^{80,81}$ Second, muscle fascicle length is routinely reported to be lower in elderly subjects than in young adults. Shorter fascicles (with fewer sarcomeres arranged in series) will generate force over a smaller range of motion and exhibit reduced maximum shortening speed, and they will also generate less force at any given rate of shortening than longer fascicles because their sarcomere shortening rates are higher. ${ }^{82}$ Third, protein muscle mass, or contractile tissue, is reduced and as a consequence muscle force is reduced. Fourth, global reduction in muscle force and shortening speed leads to a reduction in muscle power. 
Following resistance training, muscle architecture is altered in young, old, and even frail individuals: each of the architectural modifications that occurs with aging and cited above can be partially reversed with training (see Ref. ${ }^{79}$ for review). First, resistance exercise training is associated with an increase in pennation angle in old ${ }^{83}$ and even frail subjects. ${ }^{84}$ An increase in pennation angle is of benefit because more contractile material can be attached to the aponeuroses, as shown by an increase of PCSA, and it generates lateral force transmission. Although this increases the force-generating capacity of the muscle, it can simultaneously reduce the force of the muscle fibers exerted on the tendon, because the cosine of the pennation angle decreases, but the overall force exerted on the tendon remains positive as long as the pennation angle does not exceed $45^{\circ} .{ }^{79}$ Second, resistance exercise training is associated with an increase in muscle fiber length in old ${ }^{83}$ and even frail individuals, ${ }^{84}$ which would increase the shortening velocity of the muscle fiber. Third, as discussed in another section, protein muscle mass (contractile tissue) is enhanced by a resistance training program and as a consequence muscle force is increased. Fourth, global increase in muscle force and shortening speed leads to an increase in muscle power.

Aging is also associated with structural modifications of the tendons. In elderly subjects, increased tendon compliance gives a better shortening of the muscletendon complex than that measured in a stiff tendon. If one looks closely at the ascending part of the length-tension relation, the greater fascicular shortening during contraction may then contribute to the age-related reduction in specific tension. ${ }^{78}$ In a more compliant tendon, a longer time will be needed to stretch the tendon, with the result that less force will be produced over a given time. ${ }^{9}$ Two recent topical reviews indicated that the reduction in musculotendinous stiffness in older people can be mitigated by resistance training, ${ }^{85,86}$ while a walking training program over 6 months brought about increments in muscle thickness and strength in lower limbs but did not result in any changes in tendon stiffness in the elderly. 87

\section{Effect of Physical Activity on Muscle Mechanical Excitation-Contraction Coupling in Elderly Subjects}

Another factor that may account for the overall effect on the maximum shortening velocity of whole muscle in old age is the impairment of excitation-contraction $(E-C)$ coupling. ${ }^{88}$ In fact, the E-C process becomes uncoupled in old age as a consequence of a reduction in T-tubule dihydropyridine receptors and sarcoplasmic reticulum membrane receptors, such as calcium release channels and ryanodine receptors (RyR). This process may result in failure of the transduction of sarcolemmal depolarization into a calcium signal and a mechanical response. Oxidative stress, previously described as a factor that is not specifically age-related but as one that increases during the life span, could also induce qualitative alterations of sarcoplasmic reticulum function, ${ }^{89}$ in particular at the RyR level. ${ }^{90,91}$ Chronic RyR dysfunction could then be associated with (1) reduced reticular sarcoplasmic calcium release, or (2) chronic calcium leaking with a decreased calcium spike at the $\mathrm{E}-\mathrm{C}$ coupling time, or (3) an induction of the calcium-dependent apoptotic pathway. Although the effects of regular training and oxidative stress on sarcoplasmic reticulum homeostasis have never been studied in elderly subjects, this countermeasure could have a favorable influence on the E-C process in this population, as it could enhance protein synthesis and reduce oxidative stress (see the preceding sections on this topic). 


\section{PHYSICAL ACTIVITY FOR SARCOPENIA: IS THERE AN EVIDENCE-BASED EPIDEMIOLOGIC EFFECT? FROM THE CELL BACK TO HUMAN: IT'S NEVER TOO LATE!}

In addition to the beneficial effects, measurable at cell level, of a physical activity program in sarcopenia, the impact of regular physical activity in this context has also been suggested from an epidemiologic viewpoint.

According to an expert panel recently convened for a workshop of The Society for Sarcopenia, Cachexia, and Wasting Disease, exercise (both resistance and aerobic) in combination with adequate protein (leucine-enriched balanced amino acids and possibly creatine) and energy intake is the key component of the prevention and management of sarcopenia (restoring muscle mass and function), whereas adequate protein supplementation alone only slows loss of muscle mass. ${ }^{92}$ This assumption is based on evidence-based findings such as those of a recent meta-analysis that aimed to determine the effects of resistance exercise on lean body mass in older men and women, taking exercise regimens and/or age ranges into consideration. Metaregression revealed that higher volume interventions were associated with significantly greater increases in lean body mass, whereas older individuals experienced less increase. ${ }^{93,94}$ The recent prospective study by Park and colleagues ${ }^{95}$ also highlighted that after controlling data for age and/or sex, muscle mass increase over 1 year among Japanese subjects aged 65 to 84 years was associated with physical activity, more closely for the legs than for the arms, and for duration of moderate activity (>3 metabolic equivalents [METs]) than for step count. Multivariate-adjusted logistic regressions predicted that seniors who walked at least 7000 to 8000 steps per day and/or spent 15 to 20 minutes per day at an intensity of greater than 3 METs were likely to have a muscle mass above the sarcopenia threshold.

To obtain more robust evidence for the association between sarcopenia and factors such as exercise training, Patel and colleagues ${ }^{96}$ suggested that a novel approach combining epidemiologic and basic science characterization of muscle in a wellestablished birth cohort would allow an integrative investigation of functional and molecular mechanisms underlying life course influences on sarcopenia.

\section{PHYSICAL ACTIVITY FOR SARCOPENIA: HOW TO PRESCRIBE?}

Experimental and epidemiologic data have established that physical activity is useful for the primary and tertiary preventions of sarcopenia. It is therefore critical to provide subjects with guidelines to ensure safe and effective practice, just as for drug prescription. Exercise is medicine for chronic disease, and physicians need to prescribe exercise in the same manner as they prescribe drugs. ${ }^{97}$ As for any treatment (from oral recommendations to drug prescription), the medical practitioner can first provide patients with guidelines for a physical activity program if he or she is well informed about the rationale and the evidence for this approach; this step of the prescription process is knowledge. Second, the practitioner must be trained to carry out the technical part of the prescription, in particular for physical activity, a therapy implying the patient's active participation. This step of the prescription requires "know-how." Third, still because of the active dimension of the prescription and because any obstacles to physical activity must be identified (personal and environmental obstacles), the practitioner needs to develop self-awareness for social skills. Training is needed to optimize the "know-how" and self-awareness steps. The previous section provided information on the rationale for prescribing physical activity in sarcopenia. The authors now propose a schema to help practitioners standardize their prescription in this field. 
When drugs are prescribed, the pharmacologic agent has to be defined (what molecule is recommended for the disease in question?), as well as the dose (appropriate for disease severity and patient characteristics), frequency of drug intake decided (daily or weekly?), duration of treatment, and context of drug administration (does the drug need to be administrated in a medical center or not, under the control of a medical or paramedical provider or an instructor, or can the patient take it on his or her own?). When physical activity is considered as a medication, these same domains of health prescription have to be defined. This protocol provides indicators for prescribing a physical activity training program: the prescription needs to define the agent (what kind of physical activity is recommended for the disease in question), the dose to be delivered (appropriate for disease severity and patient characteristics), the frequency of physical activity sessions (whether the dose of physical activity must be taken daily or weekly), and duration and context of the training program (in or outside of a medical center but under the supervision of a health care provider or an instructor, or whether the patient can manage it on his or her own). Both patient and practitioner must be aware that a sedentary lifestyle has more side effects than physical activity.

\section{What Are the Types of Physical Activity?}

Individual or collective, indoor or outdoor, with or without a technical support, aquatic or not: these are the defining characteristics of physical activity. The choice made at the time of the prescription will take into account the safety and the efficacy of each type of activity for the disease concerned, the patient's ease of access to the activity, and his or her experience of and pleasure to be gained from it.

\section{How Can the Dose of Physical Activity be Defined?}

The dose of physical activity refers to the energy expenditure during the physical activity session. Energy expenditure is itself defined as the product of exercise intensity $\times$ exercise duration. The dose of physical activity can thus be altered by modulating exercise intensity and exercise duration.

Defining exercise intensity is a critical step, as it cannot be controlled unequivocally, whereas duration can be timed with a chronometer. Exercise intensity can be defined using subjective indicators such as a scoring scale. For example, the modified Borg Scale (subjective score ranked from 0 to 10 ) defines moderate intensity as a score from 2 to 4 and high intensity as a score from 5 to 7 . The Borg Scale can be used to measure dyspnea, muscle pain, or exercise difficulty.

Exercise intensity can also be defined using objective indicators reflecting the metabolic charge of the exercise. Any muscular contraction needs ATP hydrolysis that releases the energy necessary for sarcomeric actin-myosin cross-bridge cycling. Exercise intensity can be defined as energy output (energy quantity delivered for a time unit, $\mathrm{kcal} / \mathrm{min}$ for example). The higher the intensity of the exercise, the greater the energy supply and thus the higher the ATP output. In accordance with the kinetic properties of enzymes involved in energy metabolism, phosphagen (stored phosphocreatine and ATP) hydrolysis ensures the maximal ATP production output for muscular contraction but for a short contraction duration, whereas aerobic metabolism ensures minimal ATP production output from fatty acids and carbohydrates (and protein to a small extent) but for a very long contraction duration (lower intensity but more prolonged exercise, defining endurance exercise), and glycolytic metabolism ensures intermediate ATP production output from carbohydrates for an intermediate contraction duration. On a metabolic level, exercise intensity can be defined as low to medium for low to medium levels of energy expenditure corresponding to a low to medium level 
of recruitment by the aerobic pathway, then as high aerobic intensity and lastly as very high aerobic intensity when the flow of energy expenditure required implies increased glycolytic metabolism and phosphagen, respectively. Whatever the intensity of exercise, all metabolic pathways are recruited at the same time, but the part played by each in the total production of energy varies according to the energy flow required to sustain the muscular exercise imposed.

For aerobic exercises, exercise intensity is defined as the percentage of the maximal oxygen uptake $\left(\mathrm{V}^{\prime} \mathrm{O}_{2 m a x} ; \mathrm{V}^{\prime}\right.$ is the oxygen consumption output) sustained. For anaerobic exercises, exercise intensity is defined in relation to the maximal force. Assessing and using $\mathrm{V}^{\prime} \mathrm{O}_{2}$ to monitor exercise intensity is not suitable in the whole population because technical support is needed. However, heart rate (HR) can be used as a substitute for $\mathrm{V}^{\prime} \mathrm{O}_{2}$ and is an objective indicator for assessing aerobic exercise intensity. While power increases from rest $(R)$ during a triangular exercise, $\mathrm{V}^{\prime} \mathrm{O}_{2}$ increases linearly to exercise power up to $\mathrm{V}^{\prime} \mathrm{O}_{2 \max }$ and the corresponding maximal (M) aerobic power (AP). While $\mathrm{V}^{\prime} \mathrm{O}_{2}$ increases from $\mathrm{R}$ to $\mathrm{M}-\mathrm{AP}, \mathrm{HR}$ rises approximately linearly to $\mathrm{V}^{\prime} \mathrm{O}_{2}$ from $\mathrm{R}-\mathrm{HR}$ to $\mathrm{M}-\mathrm{HR}$. From $\mathrm{R}$ to $\mathrm{M}-\mathrm{AP}, \mathrm{V}^{\prime} \mathrm{O}_{2}$ rises from $\mathrm{R}-\mathrm{V}^{\prime} \mathrm{O}_{2}$ to $\mathrm{V}^{\prime} \mathrm{O}_{2 \max }$ and $\mathrm{HR}$ rises from $\mathrm{R}-\mathrm{HR}$ to $\mathrm{M}-\mathrm{HR}$, thus defining the heart rate reserve (HRR=M-HR minus $\mathrm{R}-\mathrm{HR}$ ). Because $\mathrm{HR}$ and $\mathrm{V}^{\prime} \mathrm{O}_{2}$ are linearly associated with exercise power under the $\mathrm{M}-\mathrm{AP}$ intensity, the relative intensity of exercise is approximately the same when defined in comparison with $\mathrm{M}-\mathrm{AP}, \mathrm{V}^{\prime} \mathrm{O}_{2 \max }$, or $\mathrm{HRR}$ for an absolute intensity. So for endurance exercises, exercise intensity can also be defined as a percentage of the HRR: low-intensity endurance exercise is defined as $25 \%$ to $44 \%$ HRR, moderate intensity as $45 \%$ to $59 \% \mathrm{HRR}$, and high intensity as more than $60 \%$ HRR (Fig. 2). Defining exercise intensity according to the percentage of HRR is suitable in a clinical population because R-HR can be checked by the physician or the patient in appropriate conditions, while $\mathrm{M}-\mathrm{HR}$ can be measured at the time of a maximal exercise test or estimated for the corresponding age $(210-0.65 \times$ age, for example). Still using cardiac frequency as an exercise intensity indicator, exercise intensity can be

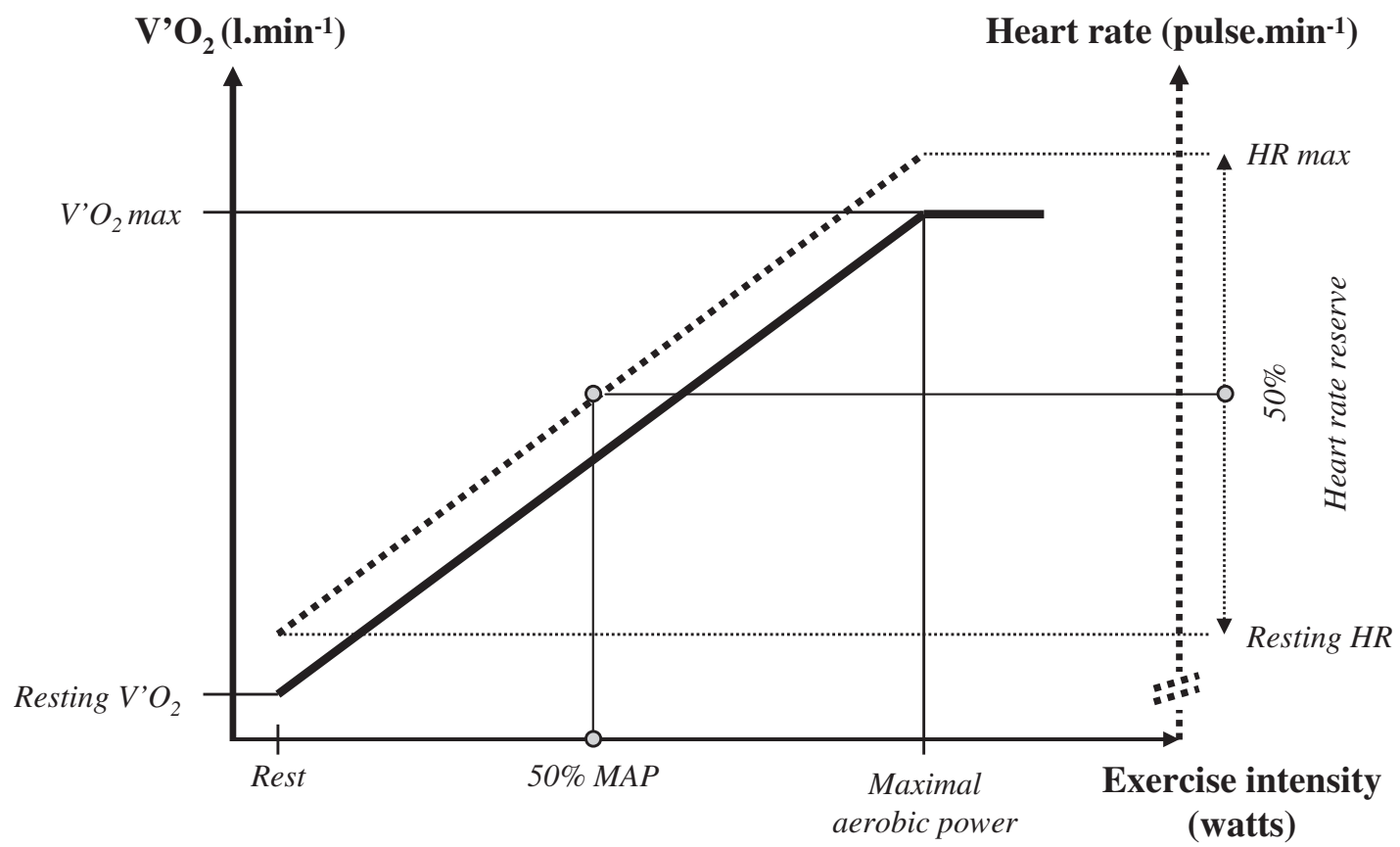

Fig. 2. Definition of endurance exercise: relationship between oxygen consumption $\left(\mathrm{V}^{\prime} \mathrm{O}_{2}\right)$, heart rate $(\mathrm{HR})$, and power $(\mathrm{P})$ during an incremental exercise testing procedure. Endurance zone is defined for any exercise intensity below $\mathrm{V}^{\prime} \mathrm{O}_{2 \max }$. Endurance exercise intensity can be derived as a percent of $\mathrm{V}^{\prime} \mathrm{O}_{2 \max }$ or heart rate reserve. MAP, maximal aerobic power. 
expressed as a percentage of M-HR: low-intensity endurance exercise is defined as $30 \%$ to $49 \% \mathrm{M}-\mathrm{HR}$, moderate intensity endurance as $50 \%$ to $69 \% \mathrm{M}-\mathrm{HR}$, and high intensity as $70 \% \mathrm{M}-\mathrm{HR}$. Although it is more direct, this method is less personalized, as it does not take into account the patient's individual resting heart rate, as HRR does. Whatever the cardiac frequency indicator used, exercise intensity can be checked with a cardiofrequency meter.

Finally, intensity can be defined for resistance exercise as a percentage of the maximum repetition test (1-RM, ie, the individual's strength level). Resistance exercise is considered for the purpose of this article from a rehabilitation viewpoint (a slow concentric phase preceded by a slow eccentric phase) and not from a metabolic viewpoint, considering resistance intensity over M-AP.

\section{How Can the Frequency of Physical Activity Sessions Be Defined?}

Regularity is a main objective of a training program for patients with chronic diseases. The frequency of physical activity sessions must be defined as days per week. When a daily physical activity dose cannot be administered in a single session, to reach the daily dose two "subdoses" can be taken in the same day, allowing a recovery time between the two. This system is useful for endurance exercise sessions that are longer than resistance sessions, and subdoses should not be less than 10 minutes.

\section{What is the Context of the Training Program?}

The context for applying the training program is defined taking into consideration the subject's cognitive capacity, his or her experience of physical activity, motivation, physical capacities, and comorbid diseases, if any. Exercise training can be performed under the control of a medical or a paramedical supervisor, in a center or not, under the supervision of an instructor or without any assistance. Environmental and ecological considerations such as personal resources, access to facilities, and a comfortable setting are central to interventions for the initiation and maintenance of the training program.

\section{Educational Therapy is Central for Exercise Prescription}

As a part of any therapeutic prescription, educational therapy is central for exercise prescription and maintenance, whatever the age of the subjects. This method is more central for positive coping strategy therapies such as physical activities. In a recent review, Winett and colleagues ${ }^{98}$ emphasized a social cognitive theory-based approach for exercise prescription in older adults. This approach highlights the importance of assisting patients to construct outcome expectancy, self-efficacy, and selfregulation of a physical activity training program. With this aim in mind, a minimal and formal set of personalized recommendations (as proposed below) are needed, while public health policy and programs could be seen as a minimum or "floor" prescription for the general population.

\section{PHYSICAL ACTIVITY FOR SARCOPENIA: WHAT EXERCISE PRESCRIPTION IS RECOMMENDED?}

Among the factors affecting a functional response of muscle to exercise training, metabolic and mechanical constraints need to be considered. The level of these constraints differs between endurance and resistance exercise training. Taking into consideration that there are hundreds of muscles of different types in the human body, each of which displays different degrees of atrophy during the aging process, it would be of interest to propose a combination of resistance and endurance training 
to activate or inhibit some major signaling mechanisms to combat age-related loss and dysfunction of muscle mass. Although combined strength and endurance training regimens can increase endurance performance as well as muscle strength and mass in comparison with strength and endurance training performed separately, ${ }^{32,99}$ it has been reported that simultaneous training for both strength and endurance results in a compromised adaptation compared with training with either exercise mode alone. $^{100}$ This effect has been variously described as the concurrent training effect or the interference effect, ${ }^{101}$ and it highlights a potential bias of combined endurance/strength training studies. This is a nonpaired dose of specific resistance and endurance exercises, as compared with specific resistance or endurance exercise training studies. To better discriminate between the significant exercise stimulatory cues leading to muscular development, a thorough mechanical-biological description of the loading condition is imperative. ${ }^{102}$ The key challenges of exercise science for sarcopenia are to best identify the most appropriate muscle-training recommendations for older adults, and to greatly increase the access to safe and effective programs in a variety of settings.

The following section presents the specific effect and value of endurance or resistance exercise in sarcopenic subjects. The authors also address other training methods suggested by recent original published research.

\section{Value of Resistance Training (and Eccentric Exercise) in Sarcopenia}

It is resistance exercise that promotes muscle hypertrophy in young and middle-aged individuals (see Ref. ${ }^{103}$ for review), and about 2 decades of age-associated loss of strength and muscle mass could be regained in about 2 months of resistance training. ${ }^{104}$ The American College of Sport Medicine and the American Heart Association have suggested that training at $70 \%$ to $90 \%$ of $1-\mathrm{RM}$ (maximal repetition) during 20 to 30 minutes on 2 or more nonconsecutive days per week is the appropriate training intensity to produce gains in muscle size and strength, even in frail elderly subjects. ${ }^{105,106}$ It has also been suggested that maintaining the benefits from resistance training in elderly people is possible with as little as one exercise session per week. ${ }^{107}$ However, as for young men, myofibrillar protein synthesis is dosedependent on intensity, and this relationship rises to a plateau at $60 \%$ to $90 \%$ of $1-\mathrm{RM}$ in older men (70 \pm 5 years), showing anabolic resistance of signaling and myofibrillar protein synthesis to resistance exercise in this population. ${ }^{20}$ Moreover, slow movements in resistance exercise are described as creating a high-intensity stimulus while decreasing the chance of injury compared with more rapid movement, ${ }^{108}$ but the optimal contraction velocity of muscles is correlated with functional capacity, and power training (force $\times$ velocity) could be a better method of improving velocity and force than concentric training. ${ }^{82}$ In summary, recommendation of resistance exercise training to promote muscle hypertrophy and strength in elderly individuals might not be appropriate for sarcopenia. The most appropriate design for a resistance training program to treat or prevent sarcopenia and to prevent physical function and mobility disability in the elderly remains to be defined, therefore more high-quality trials are needed. ${ }^{109}$

As a part of resistance exercise movements, the eccentric contraction mode could also be of specific value in regulating muscle function in elderly subjects. In fact, eccentric strength is better preserved than concentric strength in the elderly: the magnitude of preservation of eccentric strength in older adults ranges from $2 \%$ to $48 \%$, with a mean value of $21.6 \%$ from all studies, and physical inactivity is cited as a potential factor that could regulate the preservation of eccentric strength. ${ }^{81}$ Because low energy cost is coupled to high force production with eccentric exercise and 
considering this functional reserve of eccentric strength, this intervention may be useful for patients who are otherwise unable to achieve high muscle forces with traditional resistance exercise. This hypothesis has been tested in a few studies: in summary, Raj and colleagues ${ }^{82}$ recently proposed that power training and eccentric resistance training could be a better method than traditional resistance training to improve velocity and force and to limit per se muscular functional decline through the life span, but they specify that this should be tested by further targeted studies.

Apart from rehabilitation, the eccentric contraction mode could also be of value in elderly subjects for the assessment of functional capacities through the jumping mechanography method, an integrated approach to evaluation of much of the neuromusculoskeletal system response. ${ }^{110}$

\section{Value of Endurance Training for Sarcopenia}

Considering sarcopenia as a lack of muscle mass and muscle strength in older individuals, the authors assumed that resistance was the most specific type of exercise training as a countermeasure. However, it has been suggested that the association between sarcopenia and physical disability could be mediated by decreased cardiopulmonary fitness. ${ }^{111}$ Moreover, metabolic diseases that occur in the elderly are altered by endurance capacity. Because an optimal effect on the mechanical and metabolic properties of aged muscle cannot be obtained by a single type of exercise, and considering that endurance training is better than resistance training for developing endurance capacity, it has been suggested that resistance and aerobic training should be combined to combat the global issues of sarcopenia in the elderly population. ${ }^{112,113}$ Methods for codifying endurance exercise training have been described in the section "How Can the Dose of Physical Activity be Defined?," and moderate endurance training is recommended in the elderly. However, as for resistance training, the best endurance training regimen for sarcopenic subjects has still not been defined. As an original aerobic training program for sedentary aged people, aerobic interval training (AIT; weekly exercise sessions with 4-minute repetitions at a moderate aerobic intensity alternated with 1-minute repetitions at a sub-high aerobic intensity) could next be considered in sarcopenia. AIT improves aerobic capacity and M-AP in sedentary aged subjects, ${ }^{114}$ while a sub-high aerobic intensity repetitions program tends to increase the metabolic stimulus to resistance exercise in an aerobic training program.

\section{Other Training Schemes for Sarcopenia}

In recent published studies, Loenneke and colleagues ${ }^{115}$ and Fry and colleagues ${ }^{116}$ summarized the value of exercise training combined with blood flow restriction to stimulate muscle hypertrophy in older men, and provided original data. Lowintensity occlusion training as low as $20 \% 1$-RM with moderate vascular occlusion results in muscle hypertrophy in older men. Although some of the factors listed here have been identified in acute postexercise sessions (which does not prove their involvement in chronic adaptation of muscle after low-intensity occlusion training), this effect appears to work through a variety of mechanisms such as metabolic accumulation (stimulating a subsequent increase in anabolic growth factors), fast-twitch fiber recruitment, increased protein synthesis through the mTOR pathway, and modulation of ribosomal activity. Hsps, Nitric oxide synthase 1, and myostatin have also been shown to be affected by an occlusion stimulus.

The effect of vibration training on skeletal muscle has also been studied in the elderly. In an intervention study without a control group among elderly women and men (65-85 years of age), Pietrangelo and colleagues ${ }^{117}$ observed that vibration 
training could lead to enhanced maximal isometric strength with cellular and molecular adaptations, which include chronic changes in pathways related with energy metabolism, sarcomere protein balance, and oxidative stress response, but no increase in fiber or muscle. The usefulness of vibration training for sarcopenia rehabilitation needs to be determined through further standardized studies. ${ }^{118}$

\section{SUMMARY}

Evidence exists that muscle weakness, reduction in muscle mass, and reduction in physical activity occur during the life span, and lead to sarcopenia and disability in some older subjects. Evidence also exists that physical activity, which is a modifiable lifestyle behavior, can partially reverse age-associated skeletal muscle dysfunction. The links between a disease and a countermeasure, and the behavioral and environmental obstacles to access to this countermeasure for the practitioner and the patient, must be identified to optimize its prescription. While further studies are needed to identify the best qualitative and quantitative training regimen for sarcopenic subjects, the authors also propose the encouragement of "double-target" training: practitioners should be encouraged to "train" their knowledge and skill in exercise therapy through exercise prescription according to the present recommendations in order to provide good medical and practical support to sarcopenic patients for their training program.

\section{REFERENCES}

1. Muscaritoli M, Anker SD, Argiles J, et al. Consensus definition of sarcopenia, cachexia and pre-cachexia: joint document elaborated by Special Interest Groups (SIG) "cachexia-anorexia in chronic wasting diseases" and "nutrition in geriatrics". Clin Nutr 2010;29:154.

2. Cruz-Jentoft AJ, Baeyens JP, Bauer JM, et al. Sarcopenia: European consensus on definition and diagnosis: Report of the European Working Group on Sarcopenia in Older People. Age Ageing 2010;39:412.

3. Visser M, Newman AB, Nevitt MC, et al. Reexamining the sarcopenia hypothesis. Muscle mass versus muscle strength. Health, Aging, and Body Composition Study Research Group. Ann N Y Acad Sci 2000;904:456.

4. D'Antona G, Pellegrino MA, Carlizzi CN, et al. Deterioration of contractile properties of muscle fibres in elderly subjects is modulated by the level of physical activity. Eur J Appl Physiol 2007;100:603.

5. Degens H, Alway SE. Control of muscle size during disuse, disease, and aging. Int J Sports Med 2006;27:94.

6. Kortebein P, Ferrando A, Lombeida J, et al. Effect of 10 days of bed rest on skeletal muscle in healthy older adults. JAMA 2007;297:1772.

7. Lee JS, Auyeung TW, Kwok T, et al. Associated factors and health impact of sarcopenia in older Chinese men and women: a cross-sectional study. Gerontology 2007;53:404.

8. Nair KS. Aging muscle. Am J Clin Nutr 2005;81:953.

9. Reeves ND, Narici MV, Maganaris CN. Myotendinous plasticity to ageing and resistance exercise in humans. Exp Physiol 2006;91:483.

10. Bamman MM, Ragan RC, Kim JS, et al. Myogenic protein expression before and after resistance loading in 26- and 64-yr-old men and women. J Appl Physiol 2004;97:1329.

11. Kim JS, Kosek DJ, Petrella JK, et al. Resting and load-induced levels of myogenic gene transcripts differ between older adults with demonstrable sarcopenia and young men and women. J Appl Physiol 2005;99:2149. 
12. Raue U, Slivka D, Jemiolo B, et al. Myogenic gene expression at rest and after a bout of resistance exercise in young (18-30 yr) and old (80-89 yr) women. J Appl Physiol 2006;101:53.

13. Edstrom E, Ulfhake B. Sarcopenia is not due to lack of regenerative drive in senescent skeletal muscle. Aging Cell 2005;4:65.

14. Frontera WR, Reid KF, Phillips EM, et al. Muscle fiber size and function in elderly humans: a longitudinal study. J Appl Physiol 2008;105:637.

15. Buford TW, Anton SD, Judge AR, et al. Models of accelerated sarcopenia: Critical pieces for solving the puzzle of age-related muscle atrophy. Ageing Res Rev 2010;9(4):369-83.

16. Wang $X$, Proud CG. The mTOR pathway in the control of protein synthesis. Physiology (Bethesda) 2006;21:362.

17. Rivas DA, Lessard SJ, Coffey VG. mTOR function in skeletal muscle: a focal point for overnutrition and exercise. Appl Physiol Nutr Metab 2009;34:807.

18. Hulmi JJ, Tannerstedt J, Selanne H, et al. Resistance exercise with whey protein ingestion affects mTOR signaling pathway and myostatin in men. J Appl Physiol 2009;106:1720.

19. Drummond MJ, Dreyer HC, Pennings B, et al. Skeletal muscle protein anabolic response to resistance exercise and essential amino acids is delayed with aging. J Appl Physiol 2008;104:1452.

20. Kumar V, Selby A, Rankin D, et al. Age-related differences in the dose-response relationship of muscle protein synthesis to resistance exercise in young and old men. J Physiol 2009;587:211.

21. Yarasheski KE, Bhasin S, Sinha-Hikim I, et al. Serum myostatin-immunoreactive protein is increased in 60-92 year old women and men with muscle wasting. J Nutr Health Aging 2002;6:343.

22. Kosek DJ, Kim JS, Petrella JK, et al. Efficacy of 3 days/wk resistance training on myofiber hypertrophy and myogenic mechanisms in young vs. older adults. J Appl Physiol 2006;101:531.

23. Snijders T, Verdijk LB, van Loon LJ. The impact of sarcopenia and exercise training on skeletal muscle satellite cells. Ageing Res Rev 2009;8:328.

24. Vettor R, Milan G, Franzin C, et al. The origin of intermuscular adipose tissue and its pathophysiological implications. Am J Physiol Endocrinol Metab 2009;297:E987-8.

25. Koopman R, van Loon LJ. Aging, exercise, and muscle protein metabolism. J Appl Physiol 2009;106:2040.

26. Sakuma K, Yamaguchi A. Molecular mechanisms in aging and current strategies to counteract sarcopenia. Curr Aging Sci 2010;3:90.

27. Alway SE, Siu PM. Nuclear apoptosis contributes to sarcopenia. Exerc Sport Sci Rev 2008;36:51.

28. Song W, Kwak HB, Lawler JM. Exercise training attenuates age-induced changes in apoptotic signaling in rat skeletal muscle. Antioxid Redox Signal 2006;8:517.

29. Wohlgemuth SE, Seo AY, Marzetti E, et al. Skeletal muscle autophagy and apoptosis during aging: effects of calorie restriction and life-long exercise. Exp Gerontol 2010;45:138.

30. Wilkes EA, Selby AL, Atherton PJ, et al. Blunting of insulin inhibition of proteolysis in legs of older subjects may contribute to age-related sarcopenia. Am J Clin Nutr 2009;90:1343.

31. Lanza IR, Short DK, Short KR, et al. Endurance exercise as a countermeasure for aging. Diabetes 2008;57:2933. 
32. Ahtiainen JP, Hulmi JJ, Kraemer WJ, et al. Strength, endurance or combined training elicit diverse skeletal muscle myosin heavy chain isoform proportion but unaltered androgen receptor concentration in older men. Int J Sports Med 2009;30:879.

33. Kraemer WJ, Ratamess NA. Hormonal responses and adaptations to resistance exercise and training. Sports Med 2005;35:339.

34. Ratamess NA, Kraemer WJ, Volek JS, et al. Androgen receptor content following heavy resistance exercise in men. J Steroid Biochem Mol Biol 2005;93:35.

35. Spiering BA, Kraemer WJ, Vingren JL, et al. Elevated endogenous testosterone concentrations potentiate muscle androgen receptor responses to resistance exercise. J Steroid Biochem Mol Biol 2009;114:195.

36. Rahman F, Christian HC. Non-classical actions of testosterone: an update. Trends Endocrinol Metab 2007;18:371.

37. Narici MV, Maffulli N. Sarcopenia: characteristics, mechanisms and functional significance. Br Med Bull 2010;95:139.

38. Rolland Y, Czerwinski S, Abellan Van Kan G, et al. Sarcopenia: its assessment, etiology, pathogenesis, consequences and future perspectives. J Nutr Health Aging 2008;12:433.

39. Sipila S, Taaffe DR, Cheng S, et al. Effects of hormone replacement therapy and high-impact physical exercise on skeletal muscle in post-menopausal women: a randomized placebo-controlled study. Clin Sci (Lond) 2001;101:147.

40. Singh MA, Ding W, Manfredi TJ, et al. Insulin-like growth factor I in skeletal muscle after weight-lifting exercise in frail elders. Am J Physiol 1999;277:E135.

41. Hameed M, Lange $\mathrm{KH}$, Andersen $\mathrm{JL}$, et al. The effect of recombinant human growth hormone and resistance training on IGF-I mRNA expression in the muscles of elderly men. J Physiol 2004;555:231.

42. Hand BD, Kostek MC, Ferrell RE, et al. Influence of promoter region variants of insulin-like growth factor pathway genes on the strength-training response of muscle phenotypes in older adults. J Appl Physiol 2007;103:1678.

43. Karagounis LG, Hawley JA. Skeletal muscle: increasing the size of the locomotor cell. Int J Biochem Cell Biol 2010;42:1376.

44. Waters DL, Brooks WM, Qualls CR, et al. Skeletal muscle mitochondrial function and lean body mass in healthy exercising elderly. Mech Ageing Dev 2003; 124:301.

45. Melov S, Tarnopolsky MA, Beckman K, et al. Resistance exercise reverses aging in human skeletal muscle. PLoS One 2007;2:e465.

46. Lanza IR, Nair KS. Muscle mitochondrial changes with aging and exercise. Am J Clin Nutr 2009;89:467S.

47. Safdar A, Hamadeh MJ, Kaczor JJ, et al. Aberrant mitochondrial homeostasis in the skeletal muscle of sedentary older adults. PLoS One 2010;5:e10778.

48. McCully KK, Posner JD. The application of blood flow measurements to the study of aging muscle. J Gerontol A Biol Sci Med Sci 1995;50(Spec No):130.

49. Rogers MA, Evans WJ. Changes in skeletal muscle with aging: effects of exercise training. Exerc Sport Sci Rev 1993;21:65.

50. Ochi M, Kohara K, Tabara Y, et al. Arterial stiffness is associated with low thigh muscle mass in middle-aged to elderly men. Atherosclerosis 2010;212:327.

51. Payne GW. Effect of inflammation on the aging microcirculation: impact on skeletal muscle blood flow control. Microcirculation 2006;13:343.

52. Chow LS, Greenlund LJ, Asmann YW, et al. Impact of endurance training on murine spontaneous activity, muscle mitochondrial DNA abundance, gene transcripts, and function. J Appl Physiol 2007;102:1078. 
53. Cartee GD. Aging skeletal muscle: response to exercise. Exerc Sport Sci Rev 1994;22:91.

54. Brooks GA, Mercier J. Balance of carbohydrate and lipid utilization during exercise: the "crossover" concept. J Appl Physiol 1994;76:2253.

55. Hue L, Taegtmeyer H. The Randle cycle revisited: a new head for an old hat. Am J Physiol Endocrinol Metab 2009;297:E578.

56. Crentsil V. Mechanistic contribution of carnitine deficiency to geriatric frailty. Ageing Res Rev 2010;9:265.

57. Thomson DM, Brown JD, Fillmore N, et al. AMP-activated protein kinase response to contractions and treatment with the AMPK activator AICAR in young adult and old skeletal muscle. J Physiol 2009;587:2077.

58. Schrauwen P, Russell AP, Moonen-Kornips E, et al. Effect of 2 weeks of endurance training on uncoupling protein 3 content in untrained human subjects. Acta Physiol Scand 2005;183:273.

59. Gosker HR, Schrauwen P, Broekhuizen R, et al. Exercise training restores uncoupling protein-3 content in limb muscles of patients with chronic obstructive pulmonary disease. Am J Physiol Endocrinol Metab 2006;290:E976.

60. Combaret L, Dardevet D, Bechet D, et al. Skeletal muscle proteolysis in aging. Curr Opin Clin Nutr Metab Care 2009;12:37.

61. Fulle S, Di Donna S, Puglielli C, et al. Age-dependent imbalance of the antioxidative system in human satellite cells. Exp Gerontol 2005;40:189.

62. Jang YC, Lustgarten MS, Liu Y, et al. Increased superoxide in vivo accelerates age-associated muscle atrophy through mitochondrial dysfunction and neuromuscular junction degeneration. FASEB J 2010;24:1376.

63. Semba RD, Ferrucci L, Sun K, et al. Oxidative stress and severe walking disability among older women. Am J Med 2007;120:1084.

64. Ristow M, Zarse K. How increased oxidative stress promotes longevity and metabolic health: the concept of mitochondrial hormesis (mitohormesis). Exp Gerontol 2010;45:410.

65. Ji LL. Exercise-induced modulation of antioxidant defense. Ann N Y Acad Sci 2002;959:82.

66. Buford TW, Cooke MB, Willoughby DS. Resistance exercise-induced changes of inflammatory gene expression within human skeletal muscle. Eur J Appl Physiol 2009; 107:463.

67. Bautmans I, Njemini R, Vasseur S, et al. Biochemical changes in response to intensive resistance exercise training in the elderly. Gerontology 2005;51:253.

68. Wenz T, Rossi SG, Rotundo RL, et al. Increased muscle PGC-1alpha expression protects from sarcopenia and metabolic disease during aging. Proc Natl Acad Sci U S A 2009;106:20405.

69. Frisard MI, McMillan RP, Marchand J, et al. Toll-like receptor 4 modulates skeletal muscle substrate metabolism. Am J Physiol Endocrinol Metab 2010;298: E988.

70. Petersen AM, Pedersen BK. The anti-inflammatory effect of exercise. J Appl Physiol 2005;98:1154.

71. Chung HY, Cesari M, Anton S, et al. Molecular inflammation: underpinnings of aging and age-related diseases. Ageing Res Rev 2009;8:18.

72. Shaw AC, Joshi S, Greenwood H, et al. Aging of the innate immune system. Curr Opin Immunol 2010;22:507.

73. Wilkes JJ, Lloyd DJ, Gekakis N. Loss-of-function mutation in myostatin reduces tumor necrosis factor alpha production and protects liver against obesityinduced insulin resistance. Diabetes 2009;58:1133. 
74. Clark DJ, Patten C, Reid KF, et al. Impaired voluntary neuromuscular activation limits muscle power in mobility-limited older adults. J Gerontol A Biol Sci Med Sci 2010;65:495.

75. Aagaard P, Suetta C, Caserotti $P$, et al. Role of the nervous system in sarcopenia and muscle atrophy with aging: strength training as a countermeasure. Scand $\mathrm{J}$ Med Sci Sports 2010;20:49.

76. Deschenes MR, Roby MA, Eason MK, et al. Remodeling of the neuromuscular junction precedes sarcopenia related alterations in myofibers. Exp Gerontol 2010;45:389.

77. Frontera WR, Hughes VA, Krivickas LS, et al. Strength training in older women: early and late changes in whole muscle and single cells. Muscle Nerve 2003; 28:601.

78. Morse Cl, Thom JM, Reeves ND, et al. In vivo physiological cross-sectional area and specific force are reduced in the gastrocnemius of elderly men. J Appl Physiol 2005;99:1050.

79. Degens $\mathrm{H}$, Erskine RM, Morse $\mathrm{Cl}$. Disproportionate changes in skeletal muscle strength and size with resistance training and ageing. J Musculoskelet Neuronal Interact 2009;9:123.

80. Vandervoort AA. Aging of the human neuromuscular system. Muscle Nerve 2002;25:17.

81. Roig M, Macintyre DL, Eng JJ, et al. Preservation of eccentric strength in older adults: evidence, mechanisms and implications for training and rehabilitation. Exp Gerontol 2010;45:400.

82. Raj IS, Bird SR, Shield AJ. Aging and the force-velocity relationship of muscles. Exp Gerontol 2010;45:81.

83. Reeves ND, Narici MV, Maganaris CN. In vivo human muscle structure and function: adaptations to resistance training in old age. Exp Physiol 2004;89:675.

84. Suetta $C$, Andersen JL, Dalgas $U$, et al. Resistance training induces qualitative changes in muscle morphology, muscle architecture, and muscle function in elderly postoperative patients. J Appl Physiol 2008;105:180.

85. Magnusson SP, Narici MV, Maganaris CN, et al. Human tendon behaviour and adaptation, in vivo. J Physiol 2008;586:71.

86. Narici MV, Maffulli N, Maganaris CN. Ageing of human muscles and tendons. Disabil Rehabil 2008;30:1548.

87. Kubo K, Ishida Y, Suzuki S, et al. Effects of 6 months of walking training on lower limb muscle and tendon in elderly. Scand J Med Sci Sports 2008;18:31.

88. Delbono $\mathrm{O}$. Regulation of excitation contraction coupling by insulin-like growth factor-1 in aging skeletal muscle. J Nutr Health Aging 2000;4:162.

89. Galbes O, Bourret A, Nouette-Gaulain K, et al. N-acetylcysteine protects against bupivacaine-induced myotoxicity caused by oxidative and sarcoplasmic reticulum stress in human skeletal myotubes. Anesthesiology 2010;113:560.

90. Arbogast S, Beuvin M, Fraysse B, et al. Oxidative stress in SEPN1-related myopathy: from pathophysiology to treatment. Ann Neurol 2009;65:677.

91. Terentyev D, Gyorke I, Belevych AE, et al. Redox modification of ryanodine receptors contributes to sarcoplasmic reticulum $\mathrm{Ca}^{2+}$ leak in chronic heart failure. Circ Res 2008;103:1466.

92. Morley JE, Argiles JM, Evans WJ, et al. Nutritional recommendations for the management of sarcopenia. J Am Med Dir Assoc 2010;11:391.

93. Peterson MD, Sen A, Gordon PM. Influence of resistance exercise on lean body mass in aging adults: a meta-analysis. Med Sci Sports Exerc 2011;43(2): 249-58. 
94. Peterson MD, Rhea MR, Sen A, et al. Resistance exercise for muscular strength in older adults: a meta-analysis. Ageing Res Rev 2010;9:226.

95. Park H, Park S, Shephard RJ, et al. Yearlong physical activity and sarcopenia in older adults: the Nakanojo Study. Eur J Appl Physiol 2010;109:953.

96. Patel HP, Syddall HE, Martin HJ, et al. Hertfordshire sarcopenia study: design and methods. BMC Geriatr 2010;10:43.

97. Sallis RE. Exercise is medicine and physicians need to prescribe it! $\mathrm{Br} J$ Sports Med 2009;43:3.

98. Winett RA, Williams DM, Davy BM. Initiating and maintaining resistance training in older adults: a social cognitive theory-based approach. $\mathrm{Br} \mathrm{J}$ Sports Med 2009;43:114.

99. Sillanpaa E, Hakkinen A, Nyman K, et al. Body composition and fitness during strength and/or endurance training in older men. Med Sci Sports Exerc 2008; 40:950.

100. Hawley JA. Molecular responses to strength and endurance training: are they incompatible? Appl Physiol Nutr Metab 2009;34:355.

101. Nader GA. Concurrent strength and endurance training: from molecules to man. Med Sci Sports Exerc 1965;38:2006.

102. Toigo M, Boutellier U. New fundamental resistance exercise determinants of molecular and cellular muscle adaptations. Eur J Appl Physiol 2006;97:643.

103. Meng SJ, Yu LJ. Oxidative stress, molecular inflammation and sarcopenia. Int J Mol Sci 2010;11:1509.

104. Hurley BF, Roth SM. Strength training in the elderly: effects on risk factors for age-related diseases. Sports Med 2000;30:249.

105. Borst SE. Interventions for sarcopenia and muscle weakness in older people. Age Ageing 2004;33:548.

106. Nelson ME, Rejeski WJ, Blair SN, et al. Physical activity and public health in older adults: recommendation from the American College of Sports Medicine and the American Heart Association. Med Sci Sports Exerc 2007;39:1435.

107. Taaffe DR, Duret C, Wheeler S, et al. Once-weekly resistance exercise improves muscle strength and neuromuscular performance in older adults. J Am Geriatr Soc 1999;47:1208.

108. Winett RA, Carpinelli RN. Potential health-related benefits of resistance training. Prev Med 2001;33:503.

109. Chin MJ, van Uffelen JG, Riphagen I, et al. The functional effects of physical exercise training in frail older people: a systematic review. Sports Med 2008; 38:781.

110. Buehring B, Krueger D, Binkley N. Jumping mechanography: a potential tool for sarcopenia evaluation in older individuals. J Clin Densitom 2010;13:283.

111. Chien MY, Kuo HK, Wu YT. Sarcopenia, cardiopulmonary fitness, and physical disability in community-dwelling elderly people. Phys Ther 2010;90:1277.

112. Harber MP, Konopka AR, Douglass MD, et al. Aerobic exercise training improves whole muscle and single myofiber size and function in older women. Am J Physiol Regul Integr Comp Physiol 2009;297:R1452.

113. Hunter GR, McCarthy JP, Bamman MM. Effects of resistance training on older adults. Sports Med 2004;34:329.

114. Lepretre PM, Vogel T, Brechat PH, et al. Impact of a short-term aerobic interval training on maximal exercise in sedentary aged subjects. Int J Clin Pract 2009; 63:1472.

115. Loenneke JP, Wilson GJ, Wilson JM. A mechanistic approach to blood flow occlusion. Int J Sports Med 2010;31:1. 
116. Fry CS, Glynn EL, Drummond MJ, et al. Blood flow restriction exercise stimulates mTORC1 signaling and muscle protein synthesis in older men. J Appl Physiol 2010;108:1199.

117. Pietrangelo T, Mancinelli R, Toniolo L, et al. Effects of local vibrations on skeletal muscle trophism in elderly people: mechanical, cellular, and molecular events. Int J Mol Med 2009;24:503.

118. Santin-Medeiros F, Garatachea Vallejo N. Musculoskeletal effects of vibration training in the elderly. Rev Esp Geriatr Gerontol 2010;45(5):281-4 [in Spanish]. 